\title{
The ball vanishes in the air: can we blame representational momentum?
}

\author{
Cyril Thomas $^{1} \cdot$ André Didierjean $^{1}$
}

Published online: 16 May 2016

(C) Psychonomic Society, Inc. 2016

\begin{abstract}
In the vanishing ball illusion (VBI), the magician throws a ball up into the air twice and then pretends to do a third throw. On the third (fake) throw, the audience sees the ball go up and then disappear. In this article, we study the psychological mechanisms at play in this magic trick. We test the hypothesis that the illusion is based on representational momentum (RM), a psychological phenomenon in which the observer perceives the stopping point of a moving scene as being located farther ahead in the direction of motion than it really is. To determine whether the mechanisms involved in VBI are similar to those underlying RM, we compared the results of a standard VBI task to those obtained on an RM task designed to be very close to the VBI task. The results showed that VBI sensitivity was not associated with a higher anticipation score on the RM task. Unexpectedly, we found that participants who were sensitive to the illusion even obtained a weaker RM effect. We discuss several hypotheses that might account for these results.
\end{abstract}

Keywords Magic $\cdot$ Representational momentum ·

Perception $\cdot$ Illusion

Cyril Thomas

cyril.thomas@univ-fcomte.fr

André Didierjean

andre.didierjean@univ-fcomte.fr

1 University of Franche-Comté, 30 rue Mégevand, 25030 Besançon, France
For more than a thousand years, magicians have manipulated the beliefs, flaws, and limitations of the human mind to create illusions that appear to defy the laws of nature. In doing so, they have acquired a fine-grained but intuitive understanding of many facets of our cognitive functioning. The art of magic not only offers psychology an original and promising research domain for gaining insight into certain well-known cognitive processes but also may be fruitful for discovering mechanisms still poorly understood today (for reviews, see Kuhn, Amlani, \& Rensink, 2008; Kuhn \& Martinez, 2011; Macknik, MartinezConde \& Blakeslee, 2010; Macknik, Randi, Robbins, Thompson, \& Martinez-Conde, 2008; Rensink \& Kuhn, 2014; Thomas, Didierjean, Maquestiaux, \& Gygax, 2015).

One of the founders of scientific psychology, Alfred Binet (1894; see Thomas, Didierjean \& Nicolas, 2016, for a presentation of these seminal works), contended that when spectators are exposed to a magic trick, they "see what doesn't exist and don't see what does" (p. 1). Many studies (for example, those on misdirection; for more details about misdirection, see Kuhn, Caffaratti, Teszka, \& Rensink, 2014) have shown how the attention and gaze of observers can be misdirected to prevent them from seeing what exists (e.g., a secret movement; see Barnhart \& Goldinger, 2014; Kuhn \& Findlay, 2010; Kuhn \& Tatler, 2005; Kuhn, Tatler, Findlay, \& Cole, 2008; for a review, see Kuhn \& Martinez, 2011). Only a few studies have analyzed the processes that magicians manipulate to make the audience see what does not exist (Cui, Otero-Millan, Macknik, King, \& Martinez-Conde, 2011; Kuhn \& Land, 2006; Kuhn, Kourkoulou \& Leekam, 2010; Otero-Millan, Macknik, Robbins \& Martinez-Conde, 2011; Triplett, 1900). A well-known magic trick in which spectators perceive an event that does not take place is the vanishing ball illusion (VBI). 


\section{Vanishing ball illusion}

In VBI, the magician throws a ball up in the air twice, and then pretends to throw it a third time. The audience sees the ball go up and then disappear. In the early 20th century, the psychologist Norman Triplett (1900) studied this magic trick under live conditions, as he describes below:

The operator sitting behind the teacher's desk threw the ball about 3 feet in the air, catching it and letting the hand sink low behind the table. The second throw was 4 or 5 feet in height. On its return, it was dropped between the legs, but the hands went up with the regular throwing movement and were held as if awaiting the descent of the ball (p. 492).

The results of the study showed that $47 \%$ of the participants said they had "seen" the ball go up in the air on the last throw (the fake one). According to Triplett (1900), the audience's "expectations" play a major role in VBI sensitivity. Today, a century later, can we gain further insight into the psychological mechanisms involved in this trick?

A number of recent studies on VBI have dealt with the factors likely to accentuate the VBI effect (Kuhn \& Rensink, 2016; Thomas \& Didierjean, 2016). One study (Kuhn \& Land, 2006) looked at the impact of social cues, such as the magician's direction of gaze, on illusion sensitivity (for an extension of that study with autistic participants, see also Kuhn, Kourkoulou, \& Leekam, 2010). The authors created two video versions of the VBI (in these contemporaries versions, during the last fake throw, instead of lapping the ball (see Triplett, 1900), the magician secretly keeps the ball hidden in his right hand). In the first version, called "social cue pro-illusion," the magician performed the VBI while looking at the imaginary trajectory of the ball on the fake throw. In the second version, called "social cue anti-illusion," the magician did the VBI but looked instead at his right hand (the hand concealing the ball), without looking up on the fake throw. Kuhn and Land's (2006) results showed that in the proillusion condition, $68 \%$ of the participants saw the ball go up in the air on the fake throw versus $32 \%$ in the other condition. For Kuhn and Land (2006), VBI sensitivity is mediated by the magician's social cue (his gaze) ${ }^{1}$ and is not predicted by the participants' eye movements during the last fake throw. Moreover, authors stated that participants' expectations could play an important role in VBI sensitivity (for a recent

\footnotetext{
${ }^{1}$ According to Thomas and Didierjean (2016), magician social cueing is not needed in the VBI.
}

study about the role of spectators' expectations in the VBI, see Kuhn and Rensink, 2016).

Although recent studies replicated Triplett's initial findings and pointed out some of the conditions necessary for this spectacular effect to occur, the mechanisms responsible for it are still poorly understood. In the Kuhn and Land (2006) study (see also Kuhn and Rensink, 2016; Thomas, Didierjean, Maquestiaux, \& Gygax, 2015), the authors noted that one of the psychological processes that resemble this perceptual illusion the most is representational momentum (RM) (Freyd \& Finke, 1984; for reviews, see Didierjean, Ferrari, \& Blättler, 2014; Hubbard, 2005, 2015).

\section{Representational momentum}

Representational momentum refers to the tendency of observers to perceive the stopping point of a moving scene as being located farther ahead in the direction of motion than it really is (Freyd \& Finke, 1984). In a study on this effect, Hubbard and Bharucha (1988) showed participants a target (a black dot) moving slowly along a vertical or horizontal trajectory, depending on the viewing condition. After a few seconds, the target disappeared from the screen. The participants' task was to click with the computer mouse on the place where they thought the dot had disappeared. The results indicated a systematic error in the participants' responses, irrespective of the ball-trajectory condition: participants always pointed to a position ahead of the real vanishing point.

Following these studies, a large body of research showed that the RM effect was robust, and occurred systematically in a variety of contexts (e.g., a simulated plane landing, target tracking in a dynamic or static context, various auditory contexts) and could be modulated by individual factors (e.g., taskoriented attention, participant expertise) and by contextual factors (for reviews, see Didierjean, Ferrari \& Blättler, 2014; Hubbard, 2005, 2015).

\section{Is the vanishing ball illusion a question of representational momentum?}

According to Kuhn \& Rensink (2016), some similarities between the RM effect and the VBI could be highlighted. First, some studies showed that participants' expectations of the target motion can influence the RM effect (Hubbard, 1994; Hubbard \& Bharucha, 1988; Johnston \& Jones, 2006). Similarly, Kuhn and Rensink (2016) showed that VBI sensitivity is greatly influenced by knowledge of the forthcoming event (the magician will throw the ball). Second, some authors (Hubbard, 2005, 2006, 2015) assume that RM involved a joint role of top-down memory and the bottom-up perceptual components. Similar mechanisms would be involved in the VBI (Kuhn \& Rensink, 2016). Finally, the duration over which the VBI can exist (approximately 230 ms, Kuhn \& Rensink, 
2016) is similar to the duration (approximately $300 \mathrm{~ms}$ ) before RM declines (de sá Teixeira, Hecht, \& Oliveira, 2013; Freyd \& Finke, 1985; Freyd \& Johnson, 1987; Kerzel, 2000).

Although the kind of perceptual anticipation found in a VBI task resembles that involved in RM tasks, there are several major differences between the two tasks that indicate that the VBI is perhaps not due to RM (Kuhn \& Rensink, 2016). First of all, contrary to RM studies, participants in VBI studies do not anticipate a target location on a real trajectory. On the third throw in a VBI, participants "see" the ball moving along a trajectory that is expected but totally imaginary. Second, the size of the illusory forward displacement (the distance from the perceived end point to the actual end point) in a VBI task (distance from the magician's hand to the top of the screen) is indisputably greater than that identified for most representational momentum tasks (several centimeters) (Kuhn \& Rensink, 2016; Thomas, Didierjean, Maquestiaux \& Gygax, 2015). Third, nearly all participants in RM tasks are sensitive to the illusory forward displacement. This is not true of VBI tasks, where regardless of how the trick is presented (in real life or video), $68 \%$ of participants are illusion-sensitive at most. What's more, unlike RM tasks, which are not affected by trial repetition, VBI task repetition seems to eliminate the illusion (Thomas \& Didierjean, unpublished).

Despite these differences between RM and VBI tasks, an interesting question is whether and to what extent the processes involved in the two phenomena are similar. In the present article, we study the relationship between VBI sensitivity (sensitive vs. insensitive) and the anticipation score observed in an RM task. According to several studies (Blättler, Ferrari, Didierjean, Van Eslande, \& Marmèche, 2010; Blättler, Ferrari, Didierjean, \& Marmèche, 2011; Didierjean, Ferrari, \& Blättler, 2014), mechanisms responsible for the RM effect are not generic (general) but domain-specific. For example, Blättler et al.'s (2010) results showed that expert drivers exhibited a higher RM score than novice when they were exposed to a driving scene, and, for each group, their scores were not correlated to other RM scores obtained in different types of nondriving scenes (artificial or natural). These studies revealed that the RM effect is broadly influenced by individual knowledge relative to the specific category of scenes and is not necessarily transferable to other ones. Thus, if VBI sensitivity partially depends on an RM effect, we assume that an important contextual similarity between the RM task and the VBI task may increase the chances to obtain a significant transfer of the RM score from one task to the other. In this vein, we designed an RM task that was very similar to the VBI task (see the "stimuli and procedure" part). According to our hypothesis, if sensitivity to VBI is related to RM, then participants who are VBI sensitive (they "see" the ball go up in the air on the third throw) should have a higher RM score (measured as the forward displacement mean errors) than participants who are not VBI sensitive, and a positive correlation should be observed between the RM score and the strength of the VBI illusion (the ball's estimated disappearance height).

\section{Method}

\section{Participants}

Forty-two students (mean age: 20 years, SD: 1.13) from the University of Franche-Comté participated in the experiment in exchange for course credit. All participants volunteered and reported normal or corrected-to-normal vision.

\section{Stimuli and procedure}

The stimuli were displayed on a Dell Latitude E5500 comput$\mathrm{er}$, at a viewing distance of approximately $60 \mathrm{~cm}$. E-prime 2 software was used to implement the experimental procedure. The participants were tested individually, in the following two phases.

Phase 1: VBI task. We presented Kuhn and Land's (2006) social-cue-pro-illusion video version of the VBI task to all participants. In the video, the magician throws the ball up into the air twice. Then on a third, fake throw, he pretends to throw the ball up again while secretly concealing it in the palm of his right hand; with his eyes and head, he follows an imaginary ball moving upward (Fig. 1). The participants viewed the video individually, a single time. They were asked to watch it carefully and were told they were participating in an experiment about memory. They did not know they would see a magic trick. Immediately after the video, they had to use the mouse to click on the exact location where they "saw" the ball for the last time. Then, they answered a French adaptation of the Kuhn and Land (2006) ball illusion questionnaire (Appendix 1). The purpose of the questionnaire was to find out whether or not the participant had experienced the VBI. Participants were considered sensitive to the VBI when they both located the ball during the last throw above the fingers of the magician's hand (Question 1 of the ball illusion questionnaire) and verbally reported that they saw the ball moving up during the last throw (Questions 2, 4, and 5). Participants were considered not sensitive to the VBI when they located the ball during the last throw on or below the magician's hand/fingers (Question 1) or when they verbally reported that they did not see the ball moving up or going off the screen during the last throw (Questions 2, 4, and 5). Question 3 was a qualitative question that indicated 


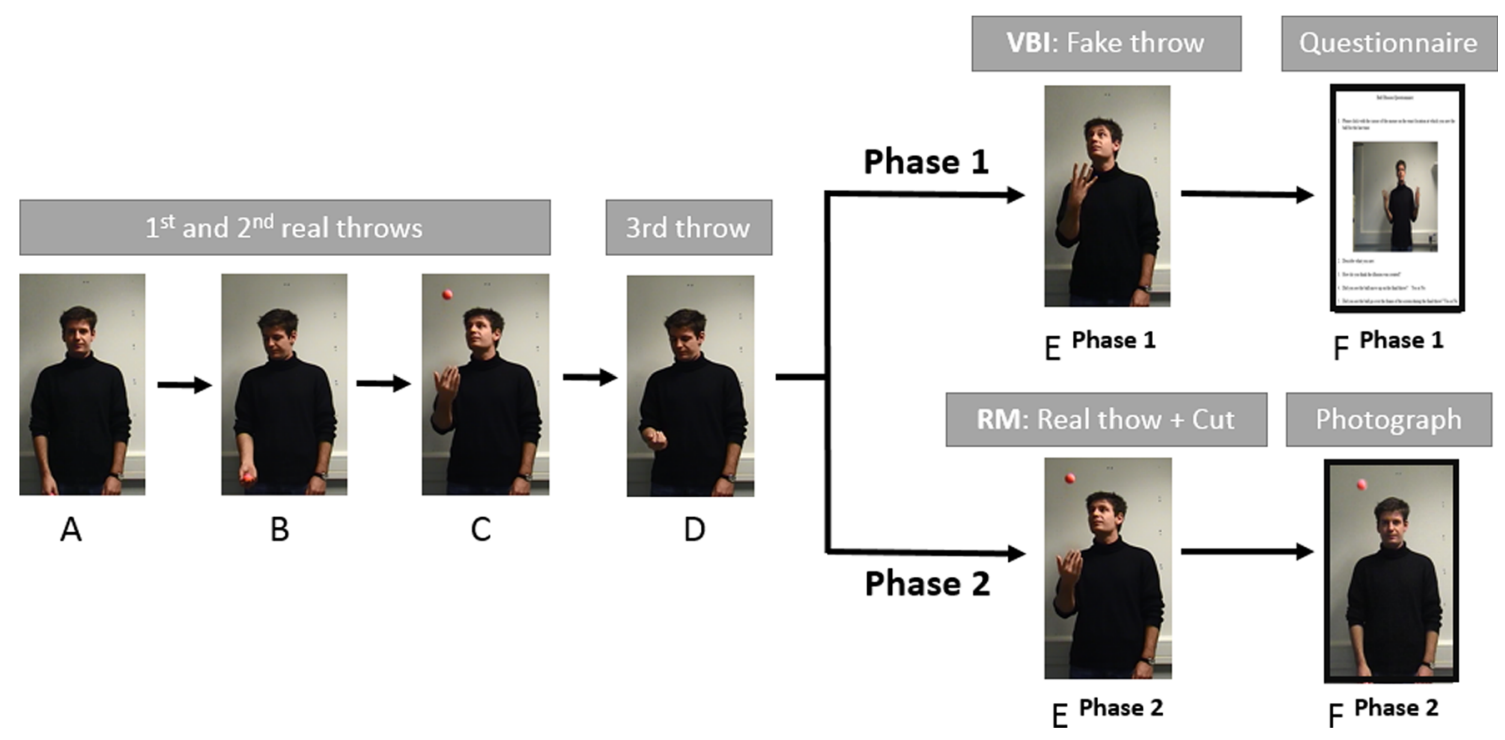

Fig. 1 Description of the two phases (VBI and RM) of Experiment 1. (a) The magician looks at the participant. (b) The magician looks at the ball held in his right hand. (c) He throws the ball up into the air, follows it with his head and eyes, and then catches it. (d) He looks at the ball held in his right hand. $\left(\mathrm{E}_{\text {phase }}\right)$ The magician pretends to throw the ball up into the air while following with his head and eyes an imaginary ball moving

whether participants sensitive to the illusion were able to, a posteriori, find the secret of the trick.

Phase 2: RM task. Phase 2 was run immediately after Phase 1. It was composed of 98 trials presented in a random order (Fig. 1). Participants were told that the first six trials were for practice only. Each trial lasted about six seconds, during which the participant viewed the same video three times in a row, without interruption. The video showed only the first real throw of the VBI task (where the magician actually throws the ball up). On the third and last viewing, the video stopped as the ball was moving upwards, and a black screen appeared in its place. For half of the trials, the video was stopped when the ball was at the height of the magician's nose; for the other half, it stopped when the ball was just above the magician's head. After this 250-ms cut (the black screen), a photograph was displayed for 3 seconds showing the magician ${ }^{2}$ and a ball in mid-air located in one of three places: in the noshift condition ( $1 / 3$ of the trials), the ball was at the same height as it had been when the video was cut; in the backward-shift condition ( $1 / 3$ of the trials), it was slightly lower (34.74 pixels or approximately $1 \mathrm{~cm})$; in the forward-shift condition ( $1 / 3$ of the trials), it was slightly higher (34.74 pixels or approximately $1 \mathrm{~cm}$ ). For each trial, the participants had to remember the height of the ball when the video stopped. Then, when they saw the photograph, they had to indicate whether the ball in the

\footnotetext{
${ }^{2}$ The magician was pictured looking straight ahead, with his arms down along the side of his body. The idea here was to avoid social cueing by the magician since it could affect the judgment task.
}

upward. $\left(\mathrm{F}_{\text {phase } 1}\right)$ Presentation of the locating task and the French version of the VBI questionnaire (Kuhn \& Land, 2006). ( $\left.\mathrm{E}_{\text {phase2 }}\right)$ The magician throws the ball up into the air and follows the ball with his head and eyes. The video is cut when the ball is in mid-air. $\left(\mathrm{F}_{\text {phase2 }}\right)$ A photograph of the magician with a ball in mid-air is displayed for the judgment task

photograph was at the same height as when the video was cut. Each trial was followed by a black screen lasting $2 \mathrm{~s}$, and then the next trial was displayed.

\section{Results}

Phase 1. The Phase-1 results replicated those obtained by Kuhn and Land (2006). Twenty-nine participants $(67.5 \%)$ were sensitive to the VBI, i.e., they said they saw the ball go up in the air on the last throw. Among these illusion-sensitive participants, 15 said they saw the ball disappear above the top of the computer screen, and 14 said they saw it disappear while still on the screen. The VBI-sensitive participants typically claimed that the illusion was created by a video montage, or by someone catching the ball above the top of the screen.

Phase 2. The results revealed an RM effect. The participants answered erroneously significantly more often in the forward-shift condition $\left(\mathrm{M}_{\text {error }}=0.67, \mathrm{SD}=0.21\right)$ than in the backward-shift condition $\left(\mathrm{M}_{\text {error }}=0.15, \mathrm{SD}=0.14\right)$, $\mathrm{t}(41)=12.05, p<0.001$. Virtually all participants $(95.24 \%)$ were sensitive to the RM effect.

Phase 2 vs. Phase 1. We compared the RM scores of the VBI-sensitive participants to those of the VBI-insensitive ones. Figure 2 gives the mean RM score for all participants, as a function of VBI sensitivity (sensitive vs. insensitive). The RM score was obtained by taking the difference between the mean error in 


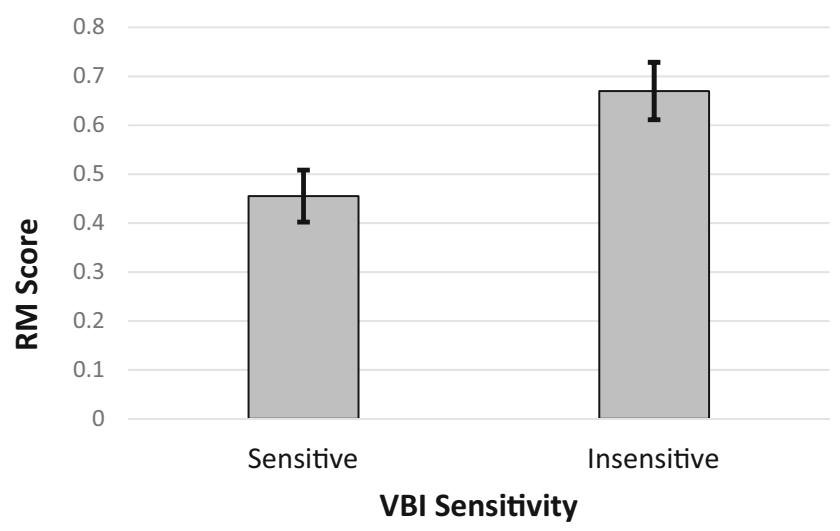

Fig. 2 Mean RM score as a function of VBI sensitivity. Error bars represent the standard error

the backward-shift condition and the mean error in the forward-shift condition. The results showed that participants who were not VBI-sensitive had a significantly higher mean RM score $(\mathrm{M}=0.67, \mathrm{SD}=0.21)$ than the sensitive ones $(\mathrm{M}=0.45, \mathrm{SD}=0.28), \mathrm{t}(40)=2.42, p<$ 0.05 (Fig. 2).

The results also showed that the mean RM score of the participants who "saw" the ball disappear on the screen $(\mathrm{M}=0.49, \mathrm{SD}=0.27)$ is not significantly different from the mean RM score of the participants who "saw" the ball disappear off-screen $(\mathrm{M}=0.42, \mathrm{SD}=$ $0.30)(\mathrm{t}(27)=0.64, p=0.53)$.

Finally, we conducted a correlation analysis to reveal the strength of the relationship between the RM score and the ball's estimated disappearance height ${ }^{3}$ (VBI task). Results of the 29 illusion-sensitive participants indicated a weak but significant negative correlation $(\mathrm{r}=-0.21, p<0.05)$ between the two variables (Fig. 3).

\section{Discussion}

VBI is a robust effect observed for approximately two-thirds of participants in most studies on this topic (Kuhn et al., 2010; Kuhn \& Land, 2006; Thomas \& Didierjean, 2016). While the VBI effect has often been demonstrated, the mechanisms that account for it are still poorly understood. One of the cognitive processes sometimes mentioned as being the closest to this perceptual illusion is representational momentum (Kuhn \& Land, 2006; Kuhn and Rensink, 2016; Thomas et al., 2015). The purpose of the present experiment was to look for a relationship between VBI and RM. We hypothesized that an elevated RM score would be accompanied by high VBI sensitivity.

\footnotetext{
${ }^{3}$ For the participants who claimed that they saw the ball disappearing offscreen, as in Kuhn and al.'s (2010) study, VBI sensitivity (in pixels) as quoted as the height of the extreme top of the computer screen.
}

Our study did not support our hypothesis. The principal results show that the illusion sensitive participants obtained a weaker RM score than those who were not sensitive. We also observed no RM difference between participants who "saw" the ball disappear on the screen and the participants who "saw" the ball disappear above the top of the screen. Finally, the correlation between VBI height estimation and RM score is weak and negative. A possible hypothesis to explain these surprising results is the existence of a variable that would act in opposite directions in the two types of task.

We assume that this variable may be related to the area where the participants directed their attention. Several studies have shown that RM declines when participants focus their attention on the moving object's trajectory and its stopping point, but increases when the observer's attention is divided or simultaneously allocated to other distractors present in the surroundings (Hayes \& Freyd, 2002; Hubbard, Kumar, \& Carp, 2009; Joordens, Spalek, Razmy, \& Van Duijn, 2004). In the VBI task, on the other hand, the effect (the number of participants sensitive to the illusion) appears to be stronger when attention is directed at the ball's imaginary movement than when it is directed toward the "suspicious" right hand of the magician that secretly conceals the ball (for the role of attentional misdirection in the VBI, see Thomas \& Didierjean, 2016). In this light, a potential hypothesis for explaining our unexpected results could be that participants who allocate more attention to the ball's real trajectory in the RM task also allocate more attention to the ball's imaginary trajectory in the VBI task. So these participants would be less sensitive in the RM task and more sensitive in the VBI task. By contrast, participants who divide their attention between the ball's trajectory (real or imaginary) and other distractors (e.g., the magician's hand) would be more sensitive in the RM task (because they pay less attention to the ball's trajectory) and less sensitive in the VBI task (because they pay less attention to the ball's imaginary trajectory and perhaps more attention to the critical distractor here, the magician's hand). In the "divided attention" hypothesis, we assume that the "magician's hand" distractor is more likely responsible for the partial attentional capture than other distractors, such as the magician's social cues (magician gaze and head direction). Indeed, according to several authors (Cui et al., 2011; OteroMillan et al., 2011; Riero, Martinez-Conde \& Macknik, 2013), magicians' hand motions could attract spectators' attention much more strongly than his gaze or head directions.

More research is needed to figure out the role of these attentional processes in the VBI. However, these future investigations could highlight some limits of eye tracking in the study of the attentional processes involved during the VBI. Indeed, in a recent eye-tracking study, Kuhn and Rensink (2016) (see also Kuhn \& Land, 2006) showed that on the fake throw in the VBI task, the illusion-sensitive participants' eye behavior did not differ from that of the insensitive ones. Thus, 
Fig. 3 VBI estimation height (in pixels) as a function of the RM score for the 29 illusionsensitive participants

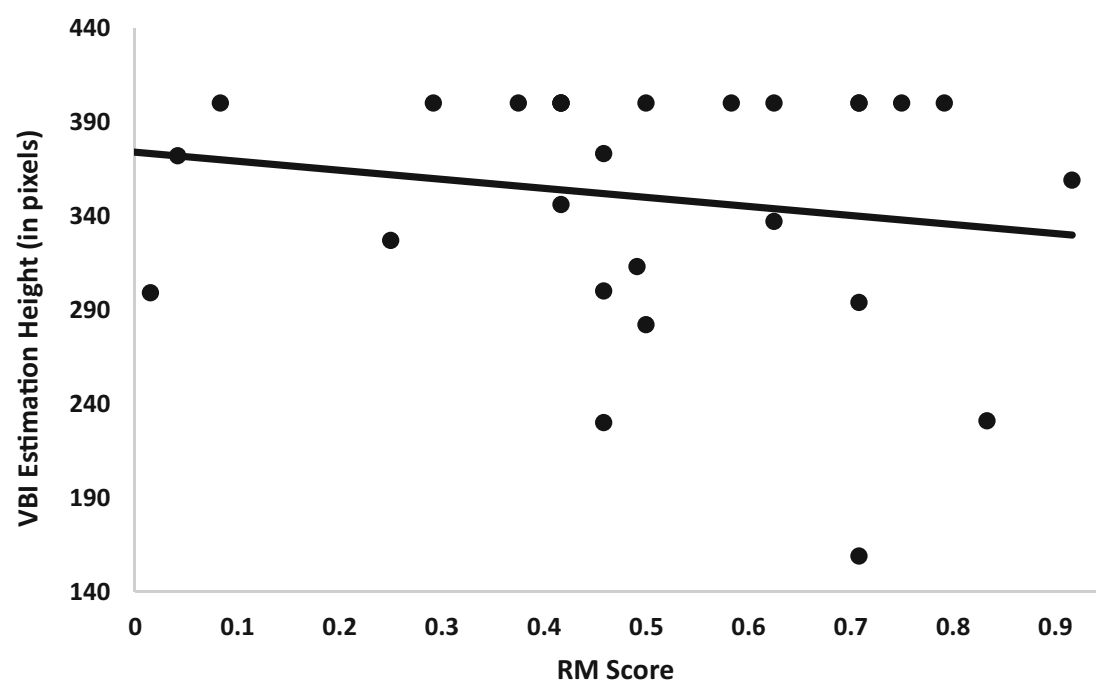

eye behavior is not a predictor of illusion sensitivity, and during a magic trick, there is sometimes a dissociation between the place where the spectators are looking and the place where they are directing their attention (Kuhn \& Findlay, 2010; Kuhn \& Tatler, 2005; Kuhn, Tatler, Findlay, \& Cole, 2008). As stated by Macknik, Randi, Robbins, Thompson and Martinez-Conde (2008), "in the more subtle "covert misdirection," the magician draws the spectator's attentional spotlight (which can be thought of as the spectator's focus of suspicion) away from the method without redirecting the spectator's gaze" (p. 872).

We assume that two other major processes could also be involved in the VBI. First, in a recent paper, Thomas and Didierjean (2016) state that the VBI sensitivity may be influenced by the way in which participants resolve the conflict between "what they saw" and "what they expected to see." For example, a participant will be more sensitive to the illusion if he prioritizes his prior expectations (the ball will move up) and if he ignores the visual feedback (the ball is gone before leaving the magician's hand). Our hypothesis is consistent with this theory. For example, participants who divided their attention in the RM task may focus less attention on the trajectory of the real object. Thus, their visual feedback of the object may be activated less and ignored more. We assume that the more the visual feedback is ignored, the more the expectations are prioritized and the higher the RM score is (for the role of the expectations on RM tasks, see Hubbard, 1994; Hubbard \& Bharucha, 1988; Johnston \& Jones, 2006). Inversely, the participants who divide their attention during the VBI task may focus a part of their attention on the magician's hand (the secret of the trick), and less attention on the "imaginary" (expected) trajectory of the ball. Thus, their visual feedback that is partly focused on the secret of the trick may be prioritized more, their

\footnotetext{
${ }^{4}$ When an observer perceives an illusory contour in image regions that contain no contrast (e.g., the Kanizsa triangle) (Singh, 2004).
}

expectations concerning the imaginary trajectory of the ball may be ignored more, and their sensitivity to the illusion may be reduced.

Second, according to Beth and Ekroll (2015) (see also Ekroll, Sayim, and Wagemans, 2013; Barnhart, 2010, Kuhn \& Rensink, 2016), one of the processes involved in the VBI could be the modal ${ }^{4}$ completion extended to the spatiotemporal domain. In this line, a perceptual completion process could be involved in the VBI, specifically when participants claimed that they saw the ball going off the screen. Indeed, when participants are exposed to the fake throw, the disappearance of the ball is an unexpected visual feedback that creates a dissonance with the expectations (the ball should move up). To resolve this dissonance, some participants may interpret the unexpected event as "the elevating ball trajectory is invisible due to its high speed and the ball has gone off the screen" (Thomas \& Didierjean, 2016). For these participants, there is a spatio-temporal gap between the initial position of the ball (into the magician's hand before the fake throw) and the imaginary ball off-screen (after the fake throw). Participants may fill the gap between these two "positions" of the ball by creating a mental image of its trajectory (by a kind of spatio-temporal modal completion). This hypothesis may explain why, in live conditions, the VBI effect is less efficient (Triplett, 1900, Thomas, unpublished). Indeed, in a live condition, participants cannot imagine the presence of the "off-screen" ball (because there is no "screen" limit) and cannot fill any gap between two spatio-temporal positions of the same object.

In conclusion, our results do not allow us to establish a simple link between VBI and RM, as some authors have suggested (Kuhn \& Land, 2006; Thomas \& Didierjean, 2016). Under our hypothesis, VBI sensitivity would not depend on a kind of RM process, but it may be influenced by the area where the spectators direct their attention on the last (fake) throw. Some other processes could be involved in the VBI, 
such as the spatio-temporal amodal completion (Beth \& Ekroll, 2015), participants' expectations (Kuhn \& Rensink, 2016) and the way in which participants resolve the conflict between "what they saw" and "what they expected to see" (Thomas \& Didierjean, 2016). Further research is needed to better understand the mechanisms responsible for the VBI, because it remains to be proven that the culprit is RM.

\section{Appendix 1}

\section{Ball illusion questionnaire}

1. Click with the mouse on the exact location at which you saw the ball for the last time.

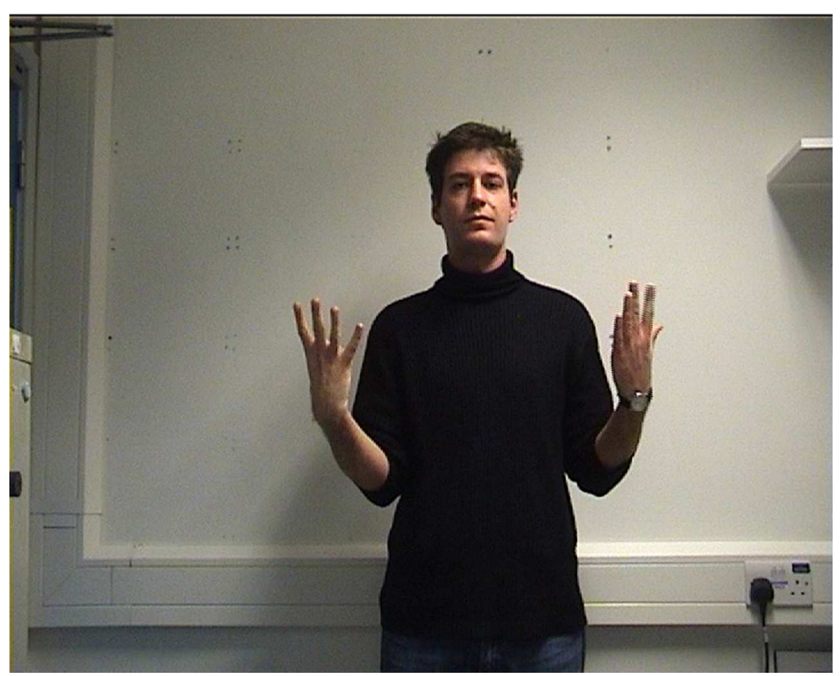

2. Describe what you saw:

3. How do you think the illusion was created?

4. Did you see the ball move up on the final throw? Yes or No

5. Did you see the ball go off the screen on the final throw? Yes or No

\section{References}

Barnhart, A. S. (2010). The exploitation of Gestalt principles by magicians. Perception, 39, 1286-1289.

Barnhart, A. S., \& Goldinger, S. D. (2014). Blinded By Magic: Eye Movements Reveal the Misdirection of Attention. Frontiers in Psychology, 5, 1461. doi: 10.3389/fpsyg.2014.01461

Beth, T., \& Ekroll, V. (2015). The curious influence of timing on the magical experience evoked by conjuring tricks involving false transfer: decay of amodal object permanence? Psychological Research, 79, 513-522. doi:10.1007/s00426014-0584-2
Binet, A. (1894). La Psychologie de la prestidigitation. Revue des Deux Mondes, 54(125), 903-923.

Blättler, C., Ferrari, V., Didierjean, A., Van Eslande, P., \& Marmèche, E. (2010). Can expertise modulate representational momentum? Visual Cognition, 18, 1253-1273.

Blättler, C., Ferrari, V., Didierjean, A., \& Marmèche, E. (2011). Representational momentum in aviation. Journal of Experimental Psychology: Human Perception and Performance, 37, 1569-1577.

Cui, J., Otero-Millan J., Macknik S. L., King M., MartinezConde, S. (2011). Social misdirection fails to enhance a magic illusion. Frontiers in Human Neuroscience, 5:103. doi: 10.3389/fnhum.2011.00103

de sá Teixeira, N., Hecht, H., \& Oliveira, A. M. (2013). The representational dynamics of remembered projectile locations. Journal of Experimental Psychology, 39, 1690-1699. doi:10.1037/a0031777

Didierjean, A., Ferrari, V., \& Blättler, C. (2014). Role of knowledge in motion extrapolation. The relevance of an approach contrasting experts and novices. Psychology of Learning and Motivation, 61, 215-235.

Ekroll, V., Sayim, B., \& Wagemans, J. (2013). Against better knowledge: Themagical force of amodal volume completion. I-Perception, 4, 511-515. doi:10.1068/i0622sas

Freyd, J. J., \& Finke, R. A. (1984). Representational momentum. Journal of Experimental Psychology: Learning, Memory, and Cognition, 10, $126-132$.

Freyd, J. J., \& Finke, R. A. (1985). A velocity effect for representational momentum. Bulletin of the Psychonomic Society, 23, 443-446. doi: 10.3758/BF03329847

Freyd, J. J., \& Johnson, J. Q. (1987). Probing the time course of representational momentum. Journal of Experimental Psychology: Learning, Memory, and Cognition, 13, 259-268. doi:10.1037/ 0278-7393.13.2.259

Hayes, A. E., \& Freyd, J. J. (2002). Representational momentum when attention is divided. Visual Cognition, 9, 8-27.

Hubbard, T. L. (1994). Judged displacement: A modular process? The American Journal of Psychology, 107, 359-373. doi:10.2307/ 1422879

Hubbard, T. L. (2005). Representational momentum and related displacements in spatial memory: A review of the findings. Psychonomic Bulletin \& Review, 12, 822-851.

Hubbard, T. L. (2006). Bridging the gap: Possible roles and contributions of representational momentum. Psicológica (Valencia), 27, 1-34.

Hubbard, T. L. (2015). The Varieties of Momentum-Like Experience. Psychological Bulletin, 141, 1081-1119. doi: 10.1037/bul0000016

Hubbard, T. L., \& Bharucha, J. J. (1988). Judged displacement in apparent vertical and horizontal motion. Perception \& Psychophysics, 44, 211-221.

Hubbard, T. L., Kumar, A. M., \& Carp, C. L. (2009). Effects of spatial cueing on representational momentum. Journal of Experimental Psychology: Learning, Memory, and Cognition, 35, 666-677.

Johnston, H. M., \& Jones, M. R. (2006). Higher order pattern structure influences auditory representational momentum. Journal of Experimental Psychology: Human Perception and Performance, 32, 2-17. doi:10.1037/0096-1523.32.1.2

Joordens, S., Spalek, T. M., Razmy, S., \& van Duijn, M. (2004). A clockwork orange: Compensation opposing momentum in memory for location. Memory \& Cognition, 32, 39-50.

Kerzel, D. (2000). Eye movements and visible persistence explain the mislocalization of the final position of a moving target. Vision Research, 40, 3703-3715. doi:10.1016/S0042-6989(00)00226-1

Kuhn, G., Amlani, A. A., \& Rensink, R. A. (2008). Towards a science of magic. Trends in Cognitive Science, 12, 349-354.

Kuhn, G., Caffaratti, H.A., Teszka, R., \& Rensink, R. A. (2014). A psychologically-based taxonomy of misdirection. Frontiers in Psychology, 5:1392. doi: 10.3389/fpsyg.2014.01392 
Kuhn, G., \& Findlay, J. M. (2010). Misdirection, attention and awareness: Inattentional blindness reveals temporal relationship between eye movements and visual awareness. The Quarterly Journal of Experimental Psychology, 63, 136-146.

Kuhn, G., Kourkoulou, A., \& Leekam, S. R. (2010). How magic changes our expectations about autism. Psychological Science, 21, 14871493. doi: $10.1177 / 0956797610383435$

Kuhn, G., \& Land, M. F. (2006). There's more to magic than meets the eye! Current Biology, 16, R950.

Kuhn, G., \& Martinez, L. M. (2011). Misdirection - past, present, and the future. Frontiers in Human Neuroscience, 5, 172.

Kuhn, G., \& Rensink, R. A. (2016). The Vanishing Ball Illusion: a new perspective on the perception of dynamic events. Cognition, 148, 64-70. doi:10.1016/j.cognition.2015.12.003

Kuhn, G., \& Tatler, B. W. (2005). Magic and fixation: Now you don't see it, now you do. Perception, 34, 1153-1161.

Kuhn, G., Tatler, B. W., Findlay, J. M., \& Cole, G. G. (2008). Misdirection in magic: Implications for the relationship between eye gaze and attention. Visual Cognition, 16, 391-405.

Macknik, S. L., Martinez-Conde, S., \& Blakeslee, S. (2010). Sleights of mind: What the neuroscience of magic reveals about our everyday deceptions. New York, NY: Henry Holt.

Macknik, S. L., Randi, J., Robbins, A., Thompson, J., \& Martinez-Conde, S. (2008). Attention and awareness in stage magic: Turning tricks into research. Nature Reviews Neuroscience, 9, 871-879.
Otero-Millan, J., Macknik, S. L., Robbins, A., \& Martinez-Conde, S. (2011). Stronger misdirection in curved than in straight motion. Frontiers in Human Neuroscience, 5, 133. doi:10.3389/fnhum. 2011.00133

Rensink, R. A., \& Kuhn, G. (2014). A Framework for Using Magic to Study the Mind. Frontiers in Psychology, 5:1508 . doi: 10.3389/ fpsyg.2014.01508

Riero, H., Martinez-Conde, S., \& Macknik, S. L. (2013). Perceptual elements in Penn \& Teller's "Cups and Balls" magic trick. Peer J, 1, 19. doi: $10.7717 /$ peerj. 19

Singh, M. (2004). Modal and amodal completion generate different shapes. Psychological Science, 15, 454-459. doi:10.1111/j.09567976.2004.00701.x

Thomas, C., \& Didierjean, A. (2016). No need for a social cue! A masked magician can also trick the audience in the vanishing ball illusion. Attention, Perception, \& Psychophysics, 78, 21-29.

Thomas, C., Didierjean, A., Maquestiaux, F., \& Gygax, P. (2015). Does magic offer a cryptozoology ground for psychology? Review of General Psychology, 19, 117-128.

Thomas, C., Didierjean, A., \& Nicolas, S. (2016). Scientific study of magic: Binet's Pioneering approach based on observation and chronophotography. American Journal of Psychology, 129, 315328.

Triplett, N. (1900). The psychology of conjuring deceptions. American Journal of Psychology, 11, 439-510. 